\title{
The gompertz exponential pareto distribution with the properties and applications to bladder cancer and hydrological datasets
}

\author{
Adewunmi Olaniran Adeyemi $^{\mathrm{a}, *}$, Eno Emmanuella Akarawak ${ }^{\mathrm{a}}$, Ismail Adedeji Adeleke ${ }^{\mathrm{b}}$ \\ ${ }^{a}$ Department of Mathematics, Faculty of Science, University of Lagos, Lagos State, Nigeria. \\ ${ }^{b}$ Department of Actuarial Science and Insurance, Faculty of Management Science, University of Lagos, Lagos State, Nigeria.
}

\section{Article history:}

Received: 23 September 2021 / Received in revised form: 17 December 2021 / Accepted: 20 December 2021

\begin{abstract}
Many existing distributions in literatures does not have the modeling fits capacity to adequately describe the real-life phenomena. The Exponential Pareto (EP) distribution has further gained some generalizations among several authors using different generator techniques with an aim to obtain a new distribution with greater flexibility. This article proposes Gompertz Exponential Pareto (GEP) distribution using the Gompertz generator. Findings from the study revealed some lifetime distributions as special cases and mathematical properties of the distribution investigated including the mean, variance, coefficient of variation, quantile, moment, moment generating function and, order statistics. The distribution can be positively or negatively skewed. It is unimodal with failure rates whose shapes could be reversed $\mathrm{J}$ bathtub, constant, decreasing and, increasing and the parameters were estimated using maximum likelihood estimation approach. Applications to two real-life datasets revealed the ability of GEP distribution to provide more flexibilities and better fit to the dataset compared to some previously proposed distributions for the data. The results also revealed that GEP had the superior performance over other generalizations of EP distribution existing in literatures and the performance has further strengthened the usefulness of the Gompertz-generator technique.
\end{abstract}

Keywords: Gompertz generator; exponential Pareto; Gompertz exponential Pareto; lifetime distributions; superior performance.

\section{Introduction}

Statisticians and researchers are interested in investigating the challenges associated with real data set that cannot be fitted with existing standard distribution due to peculiar characteristics of the population from where the data originate. The distribution generalization is a statistical process to provide flexible distributions to address the challenges and several techniques involving the combination of two or more baseline distributions discovered. Many newly developed probability distributions associated with different techniques have also flooded the literatures. Cordeiro, et al. [1] proposed the Kumaraswamy Weibull (KwW) distribution. The results obtained from recent work by Tahir et al. [2] on the New Kumaraswamy Weibull (NKwW) distribution showed that research on the generalization of probability distribution always attract an attention because of the need for quality and superior probability models that can provide the superior model fit for real-life datasets associated with environment. However, most of the existing distributions have not adequately described many important lifetime datasets such as data with heavy-tailed from the field of hydrology, material engineering, insurance, biology and health. Several authors have introduced many

\footnotetext{
* Corresponding author. Tel.: +234-701-177-8377

Email: adewunyemi@yahoo.com
}

https://doi.org/10.21924/cst.6.2.2021.551 important distributions for analyzing the real life datasets. [3] Developing the Gamma-Pareto, Alzaatreh, et al. [4] introduced Weibull Pareto. Meanwhile, Bouguignon, et al. [5] developed the Kumaraswamy Pareto and [6] introduced the Kumaraswamy Transmuted Pareto distribution, Weibull Rayleigh by Akarawak, et al. [7], Famoye, et al. [8] developed Weibull Normal, Akata [9] proposed the Weibull Logisticexponential distribution.

Exponential Pareto (EP) distribution was introduced in 2013 by Al-Kadim, et al. [10] and many generalizations of EP existing in literatures from 2015 include Transmuted Exponential Pareto (TEP) by Luguterah, et al. [11], the Kumaraswamy Exponential Pareto (KEP) by Elbatal et al. [12], Exponentiated Exponential Pareto distribution (EEPD) by Salem [13]. The Beta Exponential Pareto (BEP) distribution was proposed and studied by Aryal [14] and by Rashwan, et al. [15].

The Gompertz distribution was introduced by the late Benjamin Gompertz [16] in which the distribution was used for growth model and for fitting tumor growth mortality. The distribution characterized with monotone increasing failure rate is widely used for lifetime data in medical and reliability studies. [17] extended the distribution to the power Gompertz distribution (PGD) and the distribution became more popular after its introduction as a generator by Alizadeh, et al. [18] in the form of Gompertz-G family of distribution where $G$ was 
taken to be a baseline distribution.

Some of the existing distributions developed using the Gompertz generator can be found in Gompertz Inverse Exponential (GoIE) by Oguntunde et al. [19], and Gompertz Exponential by Adewara et al. [20]. Oguntunde et al. [21] used Frechet as the baseline distribution to develop Gompertz Frechet (GoFr). Koleoso et al. [22] developed a three parameter Gompertz Lindley distribution, and Gompertz Flexible Weibull (GoFW) was developed by Khaleel et al. [23], the Gompertz extended generalized exponential distribution by Eghwerido et al. [24], Gompertz Lomax (GoLom) by Oguntunde et al. [25], Gompertz Alpha-Power Inverted Exponential (GAPIE) distribution by Eghwerido, et al. [26]. [27] developed the Gompertz Rayleigh distribution and most recently, Ogunde et al. [28] developed Gompertz Gumbel type II (GGTT). The usefulness of these proposed distributions was investigated by way of applications to real-life datasets. [5,6] and [14] applied the distributions to the Wheaton River data while the EEP by [13], GGTT by [28] and, the (PG) distributions by [17] justified the importance of the proposed distributions by application to the bladder cancer dataset. However, the existing results can still be improved because the dataset has not received adequate model fit from the distributions. The existing results from BEP, KTP and the KP with applications to Wheaton river data revealed the need to explore for other forms of technique that can provide a better modeling fit to the data.

This research explores the possibilities of using EP as a baseline distribution for the Gompertz-G with the aim of enhancing the potentials of the components distributions. The study addresses the monotone increasing failure rate associated with Gompertz distribution with the motive of introducing a new convoluted distribution having superior goodness-of-fit for modeling heavy-tailed real-life data. The rest of the paper is arranged as follows: Section 2 describes the proposed distribution derived under materials and methods. The section also contains some important special models of the proposed distribution, the graphical visualizations of the Cumulative Distribution (CDF) and the Probability Density Functions (PDF). Section 3 discusses some properties of the distribution and Section 4 presents the estimation of parameters. Section 5 and Section 6 present the results from application to real life datasets to assess the performance of the distribution and the conclusion of this study respectively.

\section{Materials and Methods}

\subsection{The Gompertz-G Technique for Generalizing Distribution}

The cumulative distribution function of Gompertz-G family of distribution is given by

$$
F(x)=1-e^{\frac{\alpha}{\beta}\left[1-(1-G(x))^{-\beta}\right]}
$$

The corresponding density function is defined as;

$$
f(x)=\alpha g(x)(1-G(x))^{-\beta-1} e^{\frac{\alpha}{\beta}\left[1-(1-G(x))^{-\beta}\right]}
$$

$x ; \alpha, \beta>0 ; \alpha, \beta ; \alpha, \beta$ are the additional shape parameters to add flexibility to the new distribution and $G(x)$ is the baseline distribution.

\subsection{The Exponential Pareto Distribution}

The CDF of exponential Pareto for a random variable $X$ as defined by Al-Kadim et al. [10] is given by

$G(x)=1-e^{-\lambda\left(\frac{x}{k}\right)^{\theta}}$

$\theta, \lambda>0$ are the shape parameters, while $k>0$ is the scale parameter of the distribution

The density function of EP distribution is defined as,

$g(x)=\frac{\lambda \theta}{k}\left(\frac{x}{k}\right)^{\theta-1} e^{-\lambda\left(\frac{x}{k}\right)^{\theta}} ; k, \lambda, \theta>0$

$\theta, \lambda>0$ are the shape parameters while $k>0$ the scale parameter of the distribution

\subsection{The Gompertz Exponential Pareto Distribution}

The CDF of Gompertz Exponential Pareto (GEP) distribution is derived here by substituting $G(x)$ in (3) into (1) to obtain

$F(x)=1-e^{\frac{\alpha}{\beta}\left[1-\left(e^{-\lambda\left(\frac{x}{k}\right)^{\theta}}\right)^{-\beta}\right]}$

The probability density function of GEP is the derivative of the CDF in (5) and is obtained as

$f(x)=\frac{\alpha \lambda \theta}{k}\left(\frac{x}{k}\right)^{\theta-1}\left(e^{-\lambda\left(\frac{x}{k}\right)^{\theta}}\right)^{-\beta} e^{\frac{\alpha}{\beta}\left[1-\left(e^{-\lambda\left(\frac{x}{k}\right)^{\theta}}\right)^{-\beta}\right]}$

$\alpha, \beta, \theta, \lambda>0$ are the shape parameters while $k>0$ is the scale parameter of the distribution.

\subsection{Expansion of the Density Function of GEP Distribution}

Using the series expansion;

$$
e^{\frac{\alpha}{\beta}\left[1-\left(e^{-\lambda\left(\frac{x}{k}\right)^{\theta}}\right)^{-\beta}\right]}=\sum_{i=0}^{\infty} \frac{(-1)^{j}\left(\frac{\alpha}{\beta}\right)^{i}}{i !}\left(1-\left(e^{-\lambda\left(\frac{x}{k}\right)^{\theta}}\right)^{-\beta}\right)^{i}
$$

The PDF of GEP can be expressed as;

$$
\begin{aligned}
& f(x)=\frac{\alpha \lambda \theta}{k}\left(\frac{x}{k}\right)^{\theta-1}\left(e^{-\lambda\left(\frac{x}{k}\right)^{\theta}}\right)^{-\beta} \\
& \quad \times \sum_{i=0}^{\infty} \frac{(-1)^{j}\left(\frac{\alpha}{\beta}\right)^{i}}{i !}\left(1-\left(e^{-\lambda\left(\frac{x}{k}\right)^{\theta}}\right)^{-\beta}\right)^{i}
\end{aligned}
$$

Applying the binomial expansion on (5), and carrying out some algebraic additions, we obtained

$$
\begin{aligned}
& f(x)=\frac{\alpha \lambda \theta}{k}\left(\frac{x}{k}\right)^{\theta-1}\left(e^{-\lambda\left(\frac{x}{k}\right)^{\theta}}\right)^{-\beta} \\
& \times \sum_{i=0}^{\infty} \frac{\left(\frac{\alpha}{\beta}\right)^{i}}{i !} \sum_{j=0}^{\infty}(-1)^{j}\left(\begin{array}{l}
i \\
j
\end{array}\right)\left(e^{-\lambda\left(\frac{x}{k}\right)^{\theta}}\right)^{-\beta j}
\end{aligned}
$$




$$
=\frac{\alpha \lambda \theta}{k}\left(\frac{x}{k}\right)^{\theta-1} \sum_{i=0}^{\infty} \sum_{j=0}^{\infty} \frac{(-1)^{j}\left(\frac{\alpha}{\beta}\right)^{i}}{i !}\left(\begin{array}{l}
i \\
j
\end{array}\right)\left(e^{-\lambda\left(\frac{x}{k}\right)^{\theta}}\right)^{-m}
$$

where $m=\beta(j+1)$

\subsection{The Sub-models of GEP Distribution}

i. When $\lambda=1$; the GEP reduces to Gompertz Weibull distribution with CDF given by

$$
F(x)=1-e^{\frac{\alpha}{\beta}\left[1-\left(e^{-\left(\frac{x}{k}\right)^{\theta}}\right)^{-\beta}\right]}
$$

ii. When $k=\theta=1$; the GEP reduces to Gompertz Exponential distribution studied by Adewara et al. [20] with $\mathrm{CDF}$ as given by

$$
F(x)=1-e^{\frac{\alpha}{\beta}\left[1-\left(e^{-\lambda x}\right)^{-\beta}\right]}
$$

iii. When $k=1, \theta=2$, GEP reduces to Gompertz Rayleigh distribution studied by Mohammed et al. [27] with CDF given by

$$
F(x)=1-e^{\frac{\alpha}{\beta}\left[1-\left(e^{-\lambda x^{2}}\right)^{-\beta}\right]}
$$

Figure 1 shows the graphical properties showing the shapes of the GEP distribution with different arbitrary values of parameters for the shape of probability density. Figure 2 shows the cumulative distribution function.

\section{Properties of the GEP Distribution}

This section presents some of the statistical properties of Gompertz Exponential Pareto distribution under various subsections.

\subsection{Reliability Analysis}

The survival function is defined as

$$
S(x)=1-F(x)
$$

The survival function for the GEP is derived and given as

$$
S(x)=e^{\frac{\alpha}{\beta}\left[1-\left(e^{-\lambda\left(\frac{x}{k}\right)^{\theta}}\right)^{-\beta}\right]}
$$

The hazard function (failure rate) is defined as

$$
h(x)=\frac{f(x)}{1-F(x)}
$$

The hazard function for GEP is derived and given by

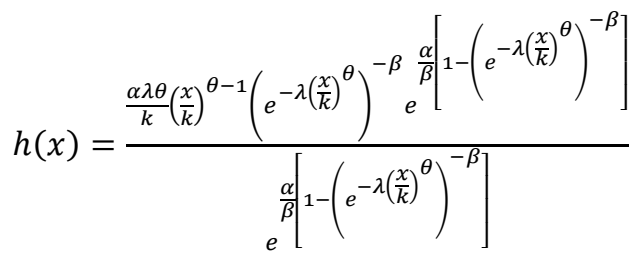

$$
h(x)=\frac{f(x)}{1-F(x)}=\frac{\alpha \lambda \theta}{k}\left(\frac{x}{k}\right)^{\theta-1}\left(e^{-\lambda\left(\frac{x}{k}\right)^{\theta}}\right)^{-\beta}
$$

The cumulative hazard function for GEP is given by

$$
-\ln (S(x))=-\frac{\alpha}{\beta}\left[1-\left(e^{-\lambda\left(\frac{x}{k}\right)^{\theta}}\right)^{-\beta}\right]
$$

The reversed hazard rate function is given by

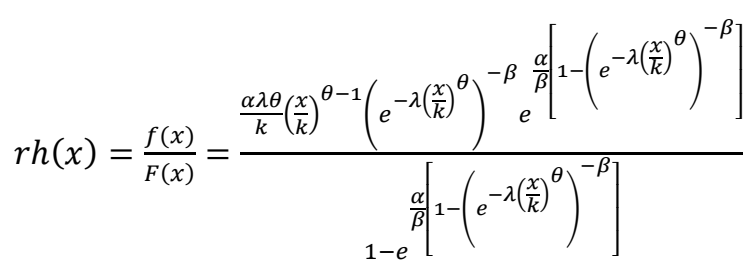

The plots of the survival and hazard rate functions of the GEP distribution for some arbitrary values of parameters are shown in figure 3 and figure 4 respectively.

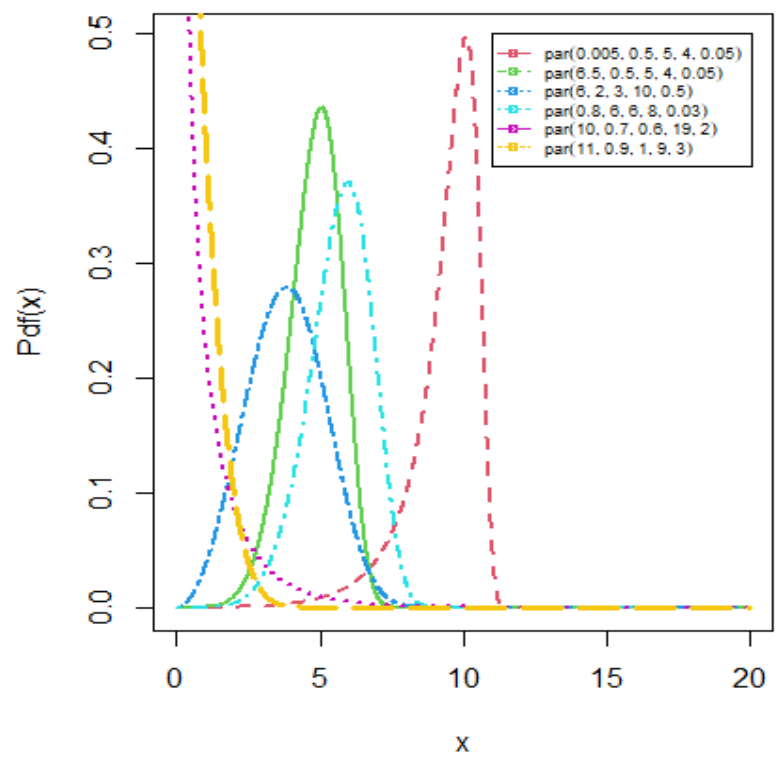

Fig. 1. The PDF of GEP distribution for some arbitrary values of the parameter

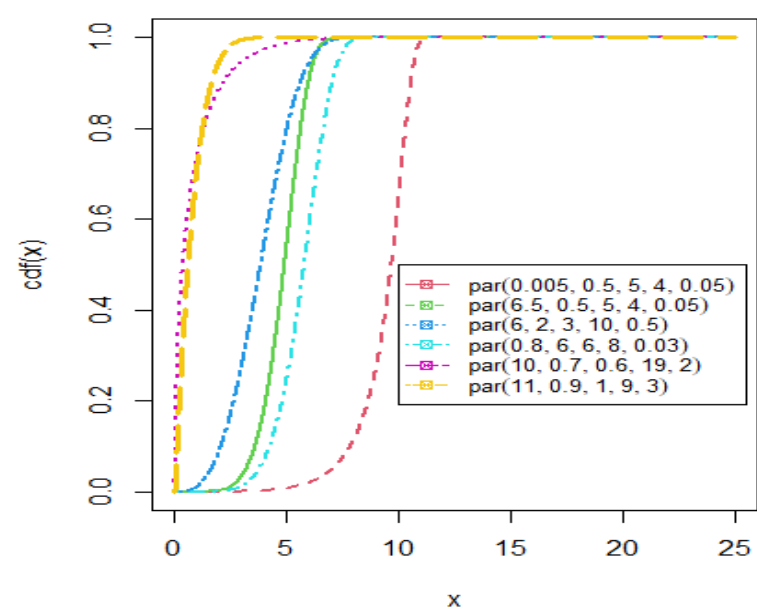

Fig. 2. The CDF of GEP distribution for some arbitrary values of the parameter 


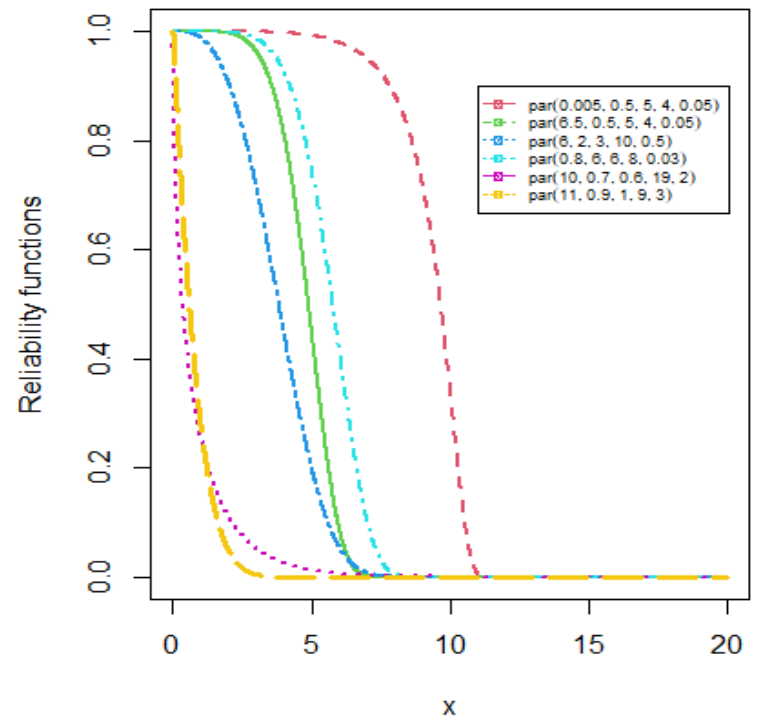

Fig. 3. The survival function for some arbitrary values of the parameter

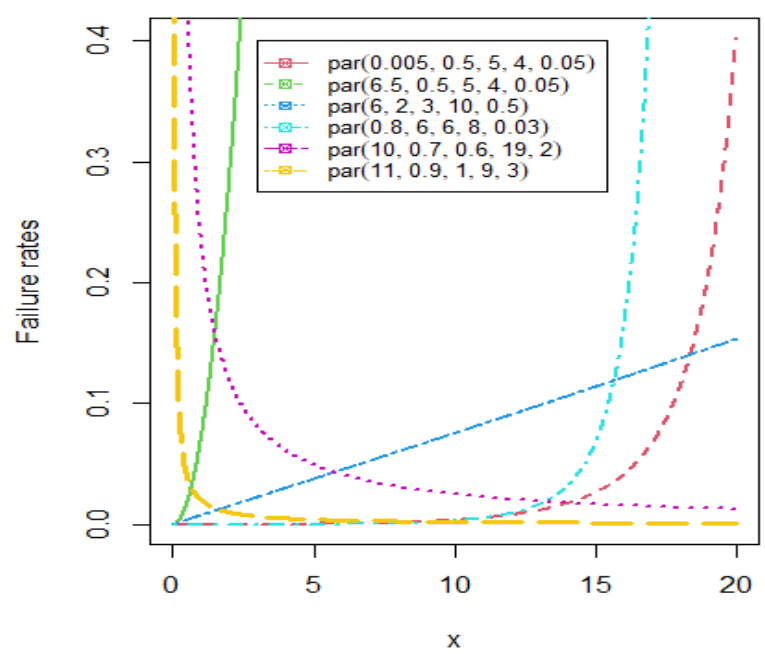

Fig. 4. The failure rates for some arbitrary values of the parameters

The characteristics of the failure (hazard) rates of GEP distribution can be summarized as follows;

i. The hazard rate is unimodal when $\theta>1$

ii. The hazard rate is $U$ bathtub shaped when $\theta<1$

iii. The GEP has the constant failure rates of $\frac{\alpha \lambda}{k}$ when $\theta=1$

iv. The hazard rate is an increasing function of $x$ when $\theta \geq 1$;

v. The hazard rate decreases when $\theta \leq 1$

vi. The GEP distribution is monotone decreasing and could become approximately symmetric as $\theta$ increases when $\theta<1$.

\subsection{The Quantile Function and Median}

Let $X$ be a random variable from the Gompertzexponential Pareto distribution whose cdf is denoted by $F(x)$ with parameters $\operatorname{GEP}(x ; \alpha, \beta, k, \lambda, \theta)$, the quantile function is defined by
$u=P\left(X \leq x_{u}\right)=F(x)$

where $\mathrm{U}$ is a uniform random variable on $\mathrm{U}(0,1)$.

The quantile for the proposed GEP distribution was obtained as

$Q(u)=k\left\{-\frac{1}{\lambda} \log \left(1-\left[1-\frac{\beta}{\alpha} \log (1-u)\right]^{-\frac{1}{\beta}}\right)\right\}^{\frac{1}{\theta}}$

Simulation was achievable by generating random samples from the GEP distribution using the random variable $X$ where we had $x=Q(u)$

The median was obtained by substituting $u=0.5$ into $Q(u)$, and the median of Gompertz-exponential Pareto is then given by

$$
x=k\left\{-\frac{1}{\lambda} \log \left(1-\left[1-\frac{\beta}{\alpha} \log (0.5)\right]^{-\frac{1}{\beta}}\right)\right\}^{\frac{1}{\theta}}
$$

\subsection{Asymptotic Behavior of GEP}

The Asymptotic properties of the Gompertz Exponential distribution was investigated by taking the limits of the density function, and hazard rate function as $x \rightarrow \infty$ and as $x \rightarrow 0$ using theorems (1 and 2).

Theorem 1: The limit of Gompertz-Exponential Pareto density function as $x \rightarrow \infty$ is 0 and as $x \rightarrow 0$ is

$\lim _{x \rightarrow 0} f(x)=\left\{\begin{array}{cc}0 & , \theta>1 \\ \frac{\alpha \lambda}{k} & , \theta=1 \\ \infty & , \theta<1\end{array}\right.$

\section{Proof:}

$\lim _{x \rightarrow 0} f(x)=\lim _{x \rightarrow 0} \frac{\alpha \lambda \theta}{k}\left(\frac{x}{k}\right)^{\theta-1}\left(e^{-\lambda\left(\frac{x}{k}\right)^{\theta}}\right)^{-\beta} e^{\frac{\alpha}{\beta}\left[1-\left(e^{-\lambda\left(\frac{x}{k}\right)^{\theta}}\right)^{-\beta}\right]}$

$\lim _{x \rightarrow 0}\left(e^{-\lambda\left(\frac{x}{k}\right)^{\theta}}\right)^{-\beta}=\lim _{x \rightarrow 0} e^{\frac{\alpha}{\beta}\left[1-\left(e^{-\lambda\left(\frac{x}{k}\right)^{\theta}}\right)^{-\beta}\right]}=1$

as $x \rightarrow 0$ when $\theta>1=0$

as $x \rightarrow 0$ when $\theta<1=\infty$

if $\theta=1$, the limit is $\frac{\alpha \lambda \theta}{k}$

$\lim _{x \rightarrow \infty} f(x)=$

$\lim _{x \rightarrow \infty} \frac{\alpha \lambda \theta}{k}\left(\frac{x}{k}\right)^{\theta-1}\left(e^{-\lambda\left(\frac{x}{k}\right)^{\theta}}\right)^{-\beta} e^{\frac{\alpha}{\beta}\left[1-\left(e^{-\lambda\left(\frac{x}{k}\right)^{\theta}}\right)^{-\beta}\right]}=0$

Theorem 2: The limit of Gompertz exponential Pareto hazard rate function as $x \rightarrow \infty$ is 0 and as $x \rightarrow 0$ is 
$\lim _{x \rightarrow 0} h(x)= \begin{cases}0 & , \theta>1 \\ \frac{\alpha \lambda}{k} & , \theta=1 \quad(24) \\ \infty & , \theta<1\end{cases}$

Proof:

The hazard rate function is given as,

$h(x)=\frac{\alpha \lambda \theta}{k}\left(\frac{x}{k}\right)^{\theta-1}\left(e^{-\lambda\left(\frac{x}{k}\right)^{\theta}}\right)^{-\beta}$

Asymptotes of hazard function rate as $x \rightarrow \infty$

$\lim _{x \rightarrow \infty} h(x)=0 ;$ when $\theta>1$
$\lim _{x \rightarrow \infty} h(x)=0 ;$ when $\theta<1$
$\lim _{x \rightarrow \infty} h(x)=0$; when $\theta=1$

Asymptotes of hazard function rate as $x \rightarrow 0$

$\lim _{x \rightarrow 0} h(x)=0 ;$ when $\theta>1$
$\lim _{x \rightarrow 0} h(x)=\infty ;$ when $\theta<1$
$\lim _{x \rightarrow 0} h(x)=\frac{\alpha \lambda \theta}{k} ;$ when $\theta=1$

Corollary 3: Let $f(x)$ and $h(x)$ be the probability density and the hazard function of Gompertz Exponential Pareto distribution respectively, then the asymptotes for $f(x)$ and $h(x)$ as $x \rightarrow 0$ imply $f(0)=h(0)$.

Proof:

Results follow from the proofs of theorems (1) and (2).

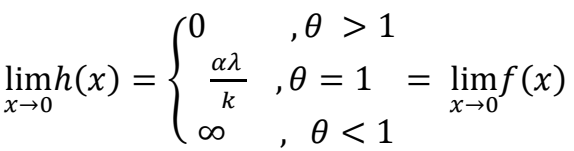

\subsection{Moments of GEP Distribution}

The moment of a random variable for continuous distribution is defined as;

$E(X)=\int_{0}^{\infty} x f(x) d x$

Theorem 3: The $r^{t h}$ moment of GEP distribution about the origin is given as follows

$$
E(X)^{r}=\sum_{i=0}^{\infty} \sum_{j=0}^{\infty} \frac{(-1)^{j}\left(\frac{\alpha}{\beta}\right)^{i}}{i !}\left(\begin{array}{l}
i \\
j
\end{array}\right) \frac{\alpha k^{r}}{m}\left(\frac{1}{\lambda m}\right)^{\frac{r}{\theta}} \Gamma\left(\frac{r}{\theta}+1\right)
$$

Proof: substitute density function of GEP in (9) into (26)

$$
\begin{aligned}
& E(X)^{r} \\
& =\int_{0}^{\infty} x^{r} \frac{\alpha \lambda \theta}{k}\left(\frac{x}{k}\right)^{\theta-1} \sum_{i=0}^{\infty} \sum_{j=0}^{\infty} \frac{(-1)^{j}\left(\frac{\alpha}{\beta}\right)^{i}}{i !}\left(\begin{array}{l}
i \\
j
\end{array}\right)\left(e^{\left.-\lambda\left(\frac{x}{k}\right)^{\theta}\right)^{m}} d x\right. \\
& =\sum_{i=0}^{\infty} \sum_{j=0}^{\infty} \frac{(-1)^{j}\left(\frac{\alpha}{\beta}\right)^{i}}{i !}\left(\begin{array}{l}
i \\
j
\end{array}\right) \int_{0}^{\infty} \frac{\alpha \lambda \theta}{k^{\theta}} x^{r+\theta-1}\left(e^{-\lambda\left(\frac{x}{k}\right)^{\theta}}\right)^{m} d x
\end{aligned}
$$

$$
\begin{aligned}
& \text { Let } \begin{aligned}
\mathrm{y} & =m \lambda\left(\frac{x}{k}\right)^{\theta}, \text { then by transformation } x=k\left(\frac{y}{\lambda m}\right)^{1 / \theta} \\
d x & =\frac{k^{\theta}}{\lambda m \theta x^{\theta-1}} d y \\
= & \sum_{i=0}^{\infty} \sum_{j=0}^{\infty} \frac{(-1)^{j}\left(\frac{\alpha}{\beta}\right)^{i}}{i !}\left(\begin{array}{l}
i \\
j
\end{array}\right) \int_{0}^{\infty} \frac{\alpha \lambda \theta}{k^{\theta}} x^{r+\theta-1} e^{-y} \frac{k^{\theta}}{\lambda m \theta x^{\theta-1}} d y \\
& =\sum_{i=0}^{\infty} \sum_{j=0}^{\infty} \frac{(-1)^{j}\left(\frac{\alpha}{\beta}\right)^{i}}{i !}\left(\begin{array}{l}
i \\
j
\end{array}\right) \int_{0}^{\infty} \frac{\alpha}{m}\left(k\left(\frac{y}{\lambda m}\right)^{1 / \theta}\right)^{r} e^{-y} d y \\
& =\sum_{i=0}^{\infty} \sum_{j=0}^{\infty} \frac{(-1)^{j}\left(\frac{\alpha}{\beta}\right)^{i}}{i !}\left(\begin{array}{l}
i \\
j
\end{array}\right) \int_{0}^{\infty} \frac{\alpha k^{r}}{m}\left(\frac{1}{\lambda m}\right)^{r / \theta} y^{r / \theta} e^{-y} d y \\
& E(X)^{r}=\sum_{i=0}^{\infty} \sum_{j=0}^{\infty} \frac{(-1)^{j}\left(\frac{\alpha}{\beta}\right)^{i}}{i !}\left(\begin{array}{l}
i \\
j
\end{array}\right) \frac{\alpha k^{r}}{m}\left(\frac{1}{\lambda m}\right)^{\frac{r}{\theta}} \Gamma\left(\frac{r}{\theta}+1\right)
\end{aligned}
\end{aligned}
$$

where $m=\beta(j+1)$

\subsection{Mean, Variance, and Coefficient of Variation of the GEP}

The mean is the first moment about the origin obtained when $r=1$ and is given by

$E(X)=\sum_{i=0}^{\infty} \sum_{j=0}^{\infty} \frac{(-1)^{j}\left(\frac{\alpha}{\beta}\right)^{i}}{i !}\left(\begin{array}{l}i \\ j\end{array}\right) \frac{\alpha k}{m}\left(\frac{1}{\lambda m}\right)^{\frac{1}{\theta}} \Gamma\left(\frac{1}{\theta}+1\right)$

The second moment is given by

$$
E\left(X^{2}\right)=\sum_{i=0}^{\infty} \sum_{j=0}^{\infty} \frac{(-1)^{j}\left(\frac{\alpha}{\beta}\right)^{i}}{i !}\left(\begin{array}{l}
i \\
j
\end{array}\right) \frac{\alpha k^{2}}{m}\left(\frac{1}{\lambda m}\right)^{\frac{2}{\theta}} \Gamma\left(\frac{2}{\theta}+1\right)
$$

The variance by definition is

$$
\operatorname{Var}(X)=E\left(X^{2}\right)-(E(x))^{2}
$$

$=\left\{\begin{array}{c}\sum_{i=0}^{\infty} \sum_{j=0}^{\infty} \frac{(-1)^{j}\left(\frac{\alpha}{\beta}\right)^{i}}{i !}\left(\begin{array}{l}i \\ j\end{array}\right) \frac{\alpha k^{2}}{m}\left(\frac{1}{\lambda m}\right)^{\frac{2}{\theta}} \Gamma\left(\frac{2}{\theta}+1\right) \\ -\left\{\sum_{i=0}^{\infty} \sum_{j=0}^{\infty} \frac{(-1)^{j}\left(\frac{\alpha}{\beta}\right)^{i}}{i !}\left(\begin{array}{l}i \\ j\end{array}\right) \frac{\alpha k}{m}\left(\frac{1}{\lambda m}\right)^{\frac{1}{\theta}} \Gamma\left(\frac{1}{\theta}+1\right)\right\}^{2}\end{array}\right.$

The coefficient of variance is defined as

$c v=\frac{\text { standard deviation }}{\text { mean }}=\frac{\sigma}{\mu}$

The coefficient of variance for GEP is given by

$$
c v=\frac{\sigma}{\sum_{i=0}^{\infty} \sum_{j=0}^{\infty} \frac{(-1)^{j}\left(\frac{\alpha}{\beta}\right)^{i}}{i !}\left(\begin{array}{l}
i \\
j
\end{array}\right) \frac{\alpha k}{m}\left(\frac{1}{\lambda m}\right)^{\frac{1}{\theta}} \Gamma\left(\frac{1}{\theta}+1\right)}
$$

where $\sigma$ is the square root of $\operatorname{Var}(X)$ in

\subsection{Moment Generating Function of GEP Distribution}

Theorem 4: Let $X$ be a five parameter Gompertz Exponential Pareto random variable with probability density 
function $g(x)$, the moment generating function of $X$ denoted as $M_{x}(t)$ is given by

$M_{X}(t)=\sum_{s, i, j=0}^{\infty} \frac{t^{s}}{s !} \frac{(-1)^{j}\left(\frac{\alpha}{\beta}\right)^{i}}{i !}\left(\begin{array}{l}i \\ j\end{array}\right) \frac{\alpha k^{r}}{m}\left(\frac{1}{\lambda m}\right)^{\frac{r}{\theta}} \Gamma\left(\frac{r}{\theta}+1\right)$

Proof:

The moment generating function for a continuous random variable $X$ is defined by

$M_{X}(t)=E\left(e^{t x}\right)=\int_{0}^{\infty} e^{t x} f(x) d x$

$E\left(e^{t x}\right)=\sum_{s=0}^{\infty} \frac{t^{s}}{s !} E\left(X^{s}\right)(34)$

$$
e^{t x}=\sum_{s=0}^{\infty} \frac{t^{s} x^{s}}{s !}
$$

By substituting the $E\left(X^{S}\right)$ in (28) into (34), the desired result for the proof was obtained

\subsection{Characteristic Function of GEP Distribution}

The characteristics function for a GEP random variable $X$ was obtained in a similar fashion as the moment generating function and is given by

$$
\varphi(i t)=\sum_{s, i, j=0}^{\infty} \frac{(-1)^{j}\left(\frac{\alpha}{\beta}\right)^{q}}{q !}\left(\begin{array}{l}
q \\
j
\end{array}\right) \frac{(i t)^{s}}{s !} \frac{\alpha k^{r}}{m}\left(\frac{1}{\lambda m}\right)^{\frac{r}{\theta}} \Gamma\left(\frac{r}{\theta}+1\right)
$$

\subsection{Order Statistics from GEP Distribution}

This sub-section is used for the derivation of $s^{\text {th }}$ order statistics and the special cases for the maximum and minimum. Suppose we have $X_{1}, X_{2}, \ldots, X_{n}$ as a random sample of size $n$ from the GEP distribution with the CDF and PDF given as $F(x)$ and $f(x)$ respectively,

let $X_{(1: n)}, X_{(2: n)}, \ldots, X_{(n: n)}$ be the appropriate order statistics designed from the arrangement of the random variables in order of magnitude, then the probability density function of the order statistics $X_{(s: n)}$ of observation in the $s^{\text {th }}$ position from the sample as defined by several authors including David and Nagaraja. [29], Arnold et al.[30] is given by

$f_{X_{s: n}}(x)=\frac{n}{(s-1) !(n-s) !} F(x)^{s-1}(1-F(x))^{n-s} f(x)$

Using the $F(x)$ and $f(x)$ in (5) and (6) respectively, the distribution of the $s^{t h}$ order statistics for Gompertz exponential Pareto is derived as

$\left\{\begin{array}{c}f_{X_{S: n}}(x)=\frac{n}{(s-1) !(n-s) !} \frac{\alpha \lambda \theta}{k}\left(\frac{x}{k}\right)^{\theta-1}\left(e^{-\lambda\left(\frac{x}{k}\right)^{\theta}}\right)^{-\beta} \\ \times e^{\frac{\alpha}{\beta}\left[1-\left(e^{-\lambda(x / k)^{\theta}}\right)^{-\beta}\right]^{n-s+1}}\left[1-e^{\left.\frac{\alpha}{\beta}\left[1-\left(e^{-\lambda(x / k)^{\theta}}\right)^{-\beta}\right]\right]^{s-1}} .\right.\end{array}\right.$

The order statistics for minimum observation is derived when $s=1$ and is given by

$f_{X_{1: n}}(x)=$

$\frac{n \alpha \lambda \theta}{k}\left(\frac{x}{k}\right)^{\theta-1}\left(e^{-\lambda\left(\frac{x}{k}\right)^{\theta}}\right)^{-\beta}\left(e^{\frac{\alpha}{\beta}\left[1-\left(e^{-\lambda(x / k)^{\theta}}\right)^{-\beta}\right]}\right)^{n}$.

The maximum order statistics is derived when $s=n$

$$
\begin{aligned}
f_{X_{n: n}}(x) & =\frac{n \alpha \lambda \theta}{k}\left(\frac{x}{k}\right)^{\theta-1}\left(e^{-\lambda\left(\frac{x}{k}\right)^{\theta}}\right)^{-\beta} e^{\frac{\alpha}{\beta}\left[1-\left(e^{-\lambda\left(\frac{x}{k}\right)^{\theta}}\right)^{-\beta}\right]} \\
& \times\left(1-e^{\frac{\alpha}{\beta}\left[1-\left(e^{-\lambda(x / k)^{\theta}}\right)^{-\beta}\right]}\right)^{n-1}
\end{aligned}
$$

\section{Estimation of Parameters of GEP Distribution}

This section is devoted to the estimation of parameters of GEP distribution using the maximum likelihood estimation (MLE) method. Let $X_{1}, X_{2}, \ldots, X_{n}$ be independent and identically distributed random sample of size $n$ from the Gompertz exponential Pareto distribution with density function $f(x)$ as given in (6) with a set of parameters $\varphi=(\alpha, \beta, k, \lambda, \theta)$.

The likelihood function of the distribution is obtained as;

$$
\begin{aligned}
& f_{X_{n: n}}(x)=\operatorname{Lik}[g(x, \varphi)]= \\
& \prod_{i=1}^{n}\left[\frac{\alpha \lambda \theta}{k}\left(\frac{x}{k}\right)^{\theta-1}\left(e^{-\lambda\left(\frac{x}{k}\right)^{\theta}}\right)^{-\beta} e^{\frac{\alpha}{\beta}\left[1-\left(e^{-\lambda\left(\frac{x}{k}\right)^{\theta}}\right)^{-\beta}\right]}\right]
\end{aligned}
$$

The $\log$ Likelihood function $\log \operatorname{Lik}[g(x, \varphi)]$ denoted as $\log L$ is

$$
\begin{aligned}
& \log L=n \log \alpha+n \log \lambda+n \log \theta-n \log k^{\theta}+(\theta-1) \sum_{1}^{n} \log x \\
&-\beta \sum_{1}^{n}\left(-\lambda\left(\frac{x}{k}\right)^{\theta}\right)+\frac{\alpha}{\beta} \sum_{1}^{n}\left[1-\left(e^{-\lambda\left(\frac{x}{k}\right)^{\theta}}\right)^{-\beta}\right]
\end{aligned}
$$

The next step is to generate the derivatives of $\log L$ with respect to the parameters

$$
\begin{aligned}
& \frac{d \log L}{d \alpha}=\frac{n}{\alpha}+\frac{n}{\beta}-\frac{1}{\beta} \sum_{1}^{n}\left(e^{-\lambda\left(\frac{x}{k}\right)^{\theta}}\right)^{-\beta} \\
& \frac{d \log L}{d \beta}=\sum_{1}^{n}\left(\lambda\left(\frac{x}{k}\right)^{\theta}\right)-\frac{n \alpha}{\beta^{2}}+\frac{\alpha}{\beta^{2}} \sum_{1}^{n}\left(e^{-\lambda\left(\frac{x}{k}\right)^{\theta}}\right)^{-\beta} \\
& -\frac{\alpha}{\beta} \sum_{1}^{n}\left(e^{-\lambda\left(\frac{x}{k}\right)^{\theta}}\right)^{-\beta} \log \left(-\lambda\left(\frac{x}{k}\right)^{\theta}\right)
\end{aligned}
$$

$\frac{d \log L}{d \lambda}=$

$\frac{n}{\lambda}+\beta \sum_{1}^{n}\left(\frac{x}{k}\right)^{\theta}-\frac{\alpha}{\beta} \sum_{1}^{n}\left(e^{-\lambda\left(\frac{x}{k}\right)^{\theta}}\right)^{-\beta-1}\left(\frac{x}{k}\right)^{\theta} e^{-\lambda\left(\frac{x}{k}\right)^{\theta}}$

$\frac{d \log L}{d \theta}=\frac{n}{\theta}-n \log k+\sum_{1}^{n} \log x+\beta \sum_{1}^{n}\left(\lambda\left(\frac{x}{k}\right)^{\theta}\right) \log \left(\frac{x}{k}\right)^{\theta}$

$-\frac{\alpha}{\beta} \sum_{1}^{n}\left(e^{-\lambda\left(\frac{x}{k}\right)^{\theta}}\right)^{-\beta} e^{-\lambda\left(\frac{x}{k}\right)^{\theta}} \log \left(\frac{x}{k}\right)^{\theta}$

$\frac{d \log L}{d k}=-\frac{n \theta}{k}+\beta \sum_{1}^{n} \theta \lambda x^{\theta} k^{-(\theta+1)}$

$+\frac{\alpha}{\beta} \sum_{1}^{n}\left(e^{-\lambda\left(\frac{x}{k}\right)^{\theta}}\right)^{-\beta-1} e^{-\lambda\left(\frac{x}{k}\right)^{\theta}} \theta \lambda x^{\theta} k^{-(\theta+1)}$ 
The parameters can be obtained by equating the normal equations to zero; however, analytical solutions are not merely prone to algebraic errors, it is also rigorous and time consuming. Hence, numerical solutions to the above equations is obtainable through statistical software. Fisher information matrix for generating variances for the confidence intervals for the estimated parameters $\hat{\varphi}=(\hat{\alpha}, \hat{\beta}, \hat{\lambda}, \hat{\theta}, \hat{k})$ can be obtained after second derivates of the normal equations

The $100(1-\epsilon) \%$ confidence intervals for parameters of GEP distribution are provided as follows;

$$
\left\{\begin{array}{cc}
\hat{\alpha} \pm Z_{\epsilon / 2} \sqrt{I_{\alpha \alpha}^{-1}(\hat{\Theta})}, & \hat{\beta} \pm Z_{\epsilon / 2} \sqrt{I_{\beta \beta}^{-1}(\hat{\Theta})} \\
\hat{\lambda} \pm Z_{\epsilon / 2} \sqrt{I^{-1}{ }_{\lambda \lambda}(\hat{\Theta})}, & , \hat{\theta} \pm Z_{\epsilon / 2} \sqrt{I^{-1} \theta \theta}(\hat{\Theta}) \\
\hat{k} \pm Z_{\epsilon / 2} \sqrt{I^{-1}{ }_{k k}(\hat{\Theta})}
\end{array}\right.
$$

where $Z_{\epsilon}$ is used as the $100(1-\epsilon) \%$ upper percentile of the standard normal distribution.

\section{Results and Discussion}

The flexibility of GEP distribution was investigated by means of application to two real-life datasets, ability of the distribution to provide a desirable model fit compared to other important and existing established distributions in literatures. The MLE of the model parameters was obtained using the AdequacyModel package in R statistical software Marinho et al. [31]. The goodness-of-fit measures for selection of the best model to the data included the Akaike Information Criteria (AIC), Bayesian Information Criteria (BIC), Consistent Akaike Information Criterion (CAIC), Hannan-Quinn Information Criteria(HQIC), the P- values, Kolgomorov Smirnoff (K-S) statistics and the Log-Likelihood $(L L)$ function. The distribution with the best performance is expected to have the lowest goodness-of-fit estimates for model selection criteria.

The Goodness-of-fit statistics are the standard model selection criteria employed by authors and researchers in making decision about competing distributions. They are contained in several related works including Khaleel et al. [23]. The goodness-of-fit statistics are defined as follows

$A I C=-2 \mathrm{~L}+2 \mathrm{~m}$

$A I C C=A I C+\frac{2 m(m+1)}{(n-m-1)}$

$C A I C=-2 L+\frac{2 m n}{(n+1)}$

$B I C=-2 \mathrm{~L}+\operatorname{mlog}(\mathrm{n})$

$H Q I C=-2 \mathrm{~L}+2 \mathrm{mlog}(\log (\mathrm{n}))$

$m$ is the number of parameters in the model and for GEP, $m=$ $5, n$ is the sample size

$L$ is the log-likelihood $L L$ function obtained from data analysis using R-Software.

The p-value and Kolmogorov Statistics are statistics corresponding to the goodness-of-fit criteria, which are the estimated values obtained from data application using Rsoftware.

\subsection{Application to Bladder Cancer Data}

Patients with bladder cancer are identified with a disease characterized with the multiplication of abnormal cells without control in the bladder. This dataset from Lee and Wang [32] is the remission (in months) of a random sample of 128 patients with bladder cancer. It has been widely applied by notable researchers to test the performance of many newly developed convoluted probability distributions including Salem [13], Elbatal et al. [33], Ateeq et al.[34], leren et al. [17] using PG, and most recently by Ogunde et al. [28] using GGTT. The GEP distribution is applied to the data and compared with EP, EEP, KEP, GGTT, GoIE, GoLom and the (PG) Power Gompertz distributions. Table 1 depicts the estimates of the parameters of the distributions.

Table 1. Maximum likelihood estimates- bladder cancer data

\begin{tabular}{lrrrrrr}
\hline \multicolumn{2}{c}{ Models $\hat{\alpha}$} & $\hat{\beta}$ & $\hat{\lambda}$ & $\hat{\theta}$ & $\hat{k}$ \\
\hline GEP & 0.8757 & -0.1477 & 0.3461 & 1.2336 & 3.1633 \\
KEP & 2.1485 & 0.7157 & 0.6263 & 0.7526 & 1.8282 \\
EEP & 3.1911 & - & 1.2545 & 0.6165 & 3.9288 \\
GGTT & 24.1803 & 0.5783 & - & 6.1335 & 0.3086 \\
GoIE & 0.0190 & 1.0088 & 0.1826 & - & - \\
GoLo & 1.5042 & 0.0199 & 0.3563 & 3.8073 & - \\
EP & - & - & 0.9349 & 1.0591 & 8.8323 \\
PGD & 0.0950 & 0.0024 & - & 1.0377 & - \\
\hline
\end{tabular}

The estimated goodness-of-fit statistics for the selection of distribution with the best performance in modeling the data are presented in table 2 .

Table 2. Goodness-of-fit estimates for bladder cancer data

\begin{tabular}{lccccc}
\hline Models & LL & AIC & BIC & K-S & P-value \\
\hline GEP & 410.82 & 831.63 & 845.89 & 0.048 & 0.9248 \\
KEP & 410.92 & 831.86 & 846.12 & 0.051 & 0.8916 \\
EEP & 410.94 & 829.63 & 841.04 & 0.052 & 0.8741 \\
GGTT & 413.59 & 835.18 & 846.58 & 0.058 & 0.7837 \\
GoIE & 413.63 & 833.26 & 841.82 & 0.076 & 0.4520 \\
GoLo & 413.92 & 835.85 & 847.26 & 0.095 & 0.2013 \\
EP & 414.18 & 834.26 & 842.82 & 0.069 & 0.5841 \\
PG & 414.82 & 835.62 & 844.18 & 0.071 & 0.5321 \\
\hline
\end{tabular}

Table 2 reveals that the GEP has the highest $\mathrm{P}$-value with corresponding smallest values of goodness of fit measures and the result has shown that the new distribution has the capability of fitting the data more than the other distributions. 
The graphical display of the estimated density and CDF plots for the competing distributions with application to the bladder cancer data are shown in figure 5 and figure 6 respectively.

\section{Estimated Densities}

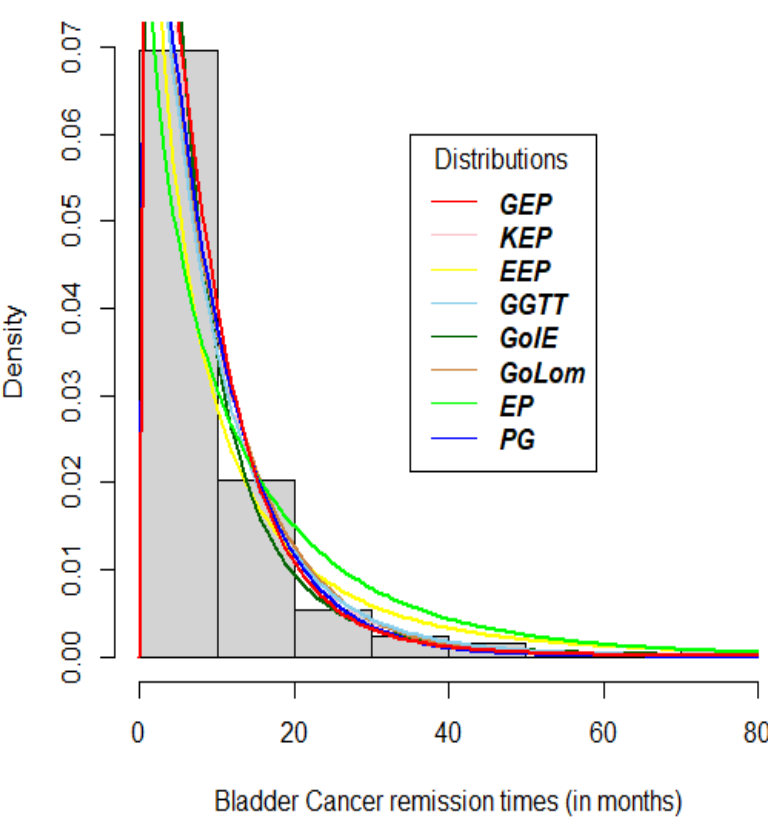

Fig. 5. The fitted densities of GEP with some competing models Estimated cdfs

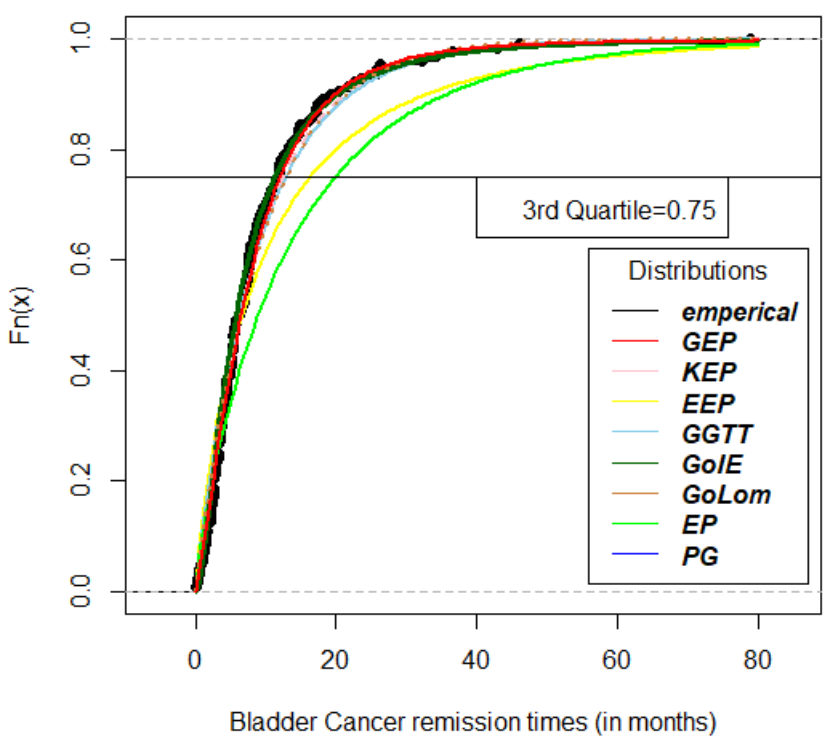

Fig. 6. The fitted cdfof GEP with some competing models

Figure 5 depicts the density plot and figure 6 shows the CDF plots of the competing models, which also supports the results from the computations in table 3 that GEP is the best choice among the distributions for modeling the dataset.

\subsection{Application to flood peaks of the Wheaton River data}

The second dataset represents 72 exceedances for the period of 1958-1984 of flood peaks (in $\mathrm{m}^{3} / \mathrm{s}$ ) of the Wheaton River near Carcross in Yukon Territory, Canada. The data is available in many of the literatures where it has been applied including Bourguignon et al. [5] using KP, Chhetri et al. [6] using KTP and Aryal [14] using BEP distributions. Table 3 contains the values of estimated parameters.

Table 3. Maximum likelihood estimates-Wheaton river data

\begin{tabular}{|c|c|c|c|c|c|}
\hline Models & $\hat{\alpha}$ & $\hat{\beta}$ & 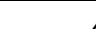 & $\widehat{\theta}$ & $\widehat{k}$ \\
\hline GEP & 14.6439 & 5.7435 & 0.0063 & 0.7422 & 0.5941 \\
\hline TEP & 0.8779 & - & 0.7726 & 0.6829 & 31.6923 \\
\hline WEP(new) & 0.5172 & - & 0.0167 & 1.6831 & 0.9854 \\
\hline KEP & 0.8596 & 0.7174 & 0.4998 & 0.8983 & 3.7821 \\
\hline BEP & 0.5482 & 0.4984 & 0.0332 & 1.2985 & 0.7474 \\
\hline EEP & 1.7893 & - & 0.2104 & 0.8919 & 1.9940 \\
\hline $\mathrm{EP}$ & - & - & 0.1790 & 0.8649 & 1.8640 \\
\hline KTP & 9.4442 & 74.899 & 0.0012 & 0.0039 & 0.1278 \\
\hline $\mathrm{KP}$ & 18.695 & 8.5151 & - & 0.0027 & 0.2680 \\
\hline
\end{tabular}

Table 4 shows the estimated values of goodness-of-fit measures for selecting the best model. The result in table 4 showed that GEP distribution had the lowest goodness-of-fit measures and the highest p-value with corresponding Kolmogorov Smirnoff test statistics.

\begin{tabular}{lccccc}
\multicolumn{6}{l}{ Table 4. Goodness-of-fits estimates for Wheaton river data } \\
\hline Models & LL & AIC & BIC & K-S & P-value \\
\hline GEP & 249.89 & 509.77 & 521.16 & 0.095 & 0.5307 \\
WEP (new) & 250.57 & 509.13 & 518.23 & 0.103 & 0.4348 \\
TEP & 250.71 & 509.44 & 518.55 & 0.195 & 0.0086 \\
KEP & 250.72 & 511.56 & 522.94 & 0.105 & 0.4038 \\
BEP & 250.98 & 511.96 & 523.34 & - & - \\
EEP & 251.35 & 511.50 & 520.60 & 0.107 & 0.3792 \\
EP & 251.47 & 508.94 & 515.77 & 0.136 & 0.1374 \\
KTP & 253.97 & 517.94 & 529.32 & 0.131 & 0.1723 \\
KP & 256.43 & 520.87 & 529.97 & 0.135 & 0.1471
\end{tabular}

Figure 7 and figure 8 represent the graphical display of the estimated density and CDF plots respectively for the competing distributions with an application to the Wheaton river data. The graphical visualization from the plots of estimated density in figure 7 and $C D F$ in figure 8 strengthened the result depicted in table 4 showing that GEP distribution is the best choice for modeling the flood peaks dataset due to the superior goodnessof-fit statistics.

\section{Conclusion}

This article develops the Gompertz Exponential Pareto (GEP) distribution as a new generalization of EP model. The distribution generalizes some lifetime distributions existing in literatures. Several mathematical properties of the new distribution were investigated. Unlike the Gompertz distribution, which is characterized by a monotone increasing 
failure rate, the GEP distribution possesses a flexible characteristic that makes it suitable for modeling phenomena with constant failure rates, decreasing and increasing failure rates.

\section{Estimated Densities}

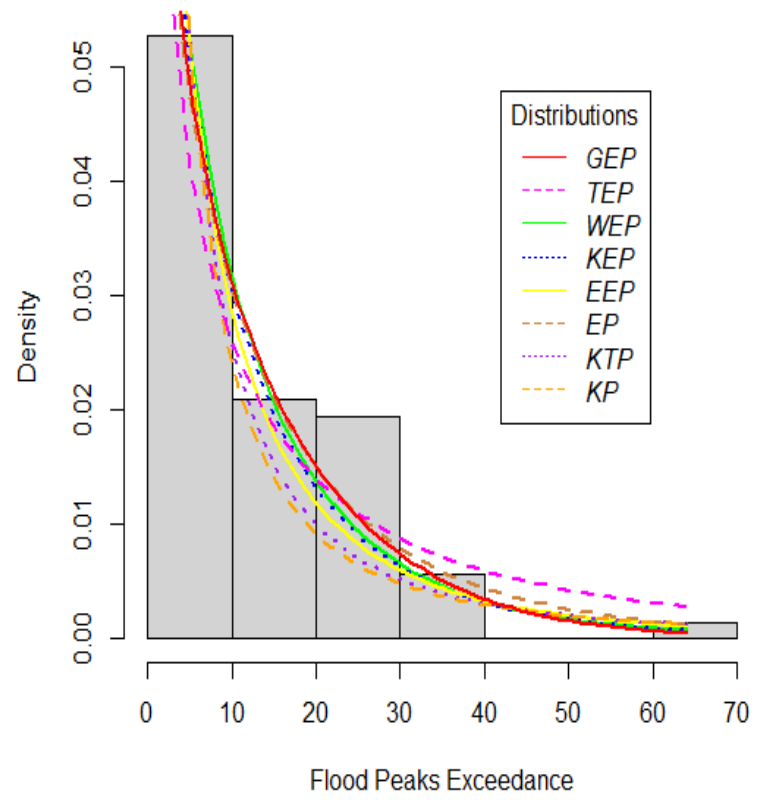

Fig. 7. The fitted densities of GEP with some competing models

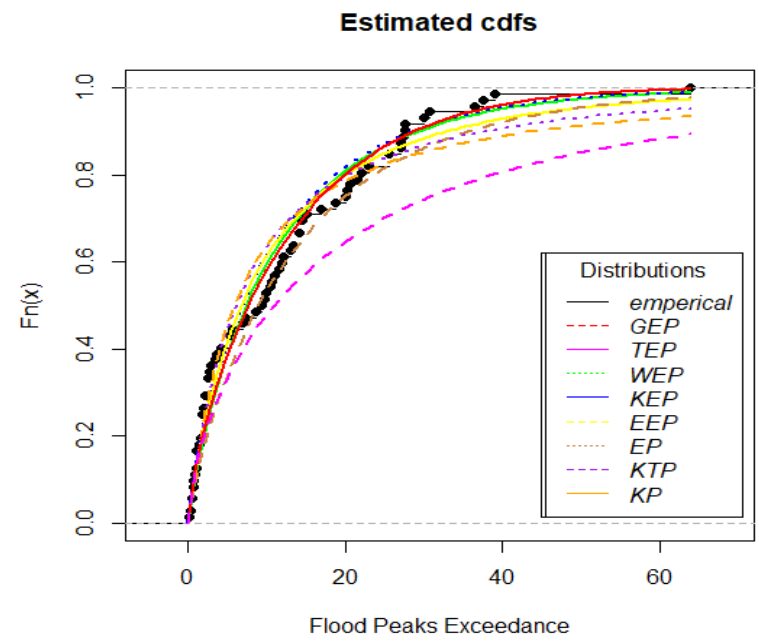

Fig. 8. The fitted densities of GEP with some competing models

The usefulness of the proposed distribution for modeling the heavy-tailed random phenomena was explored by applications to two real-life datasets from the field of medicine and hydrology. Results from data analysis revealed the superior performance of the GEP distribution with the highest goodnessof-fit model selection criteria for describing the real-life datasets. The potentials of the proposed distribution make it a good choice in several other areas of applications such as in analyzing and modeling risks associated with heavy-tailed transactions in actuarial and insurance, finance, medicine, and some extreme events in meteorology and epidemiology. The mean and variance of the GE distribution by Adewara et al. [20] were obtained in an integral form; however, the results from GEP had the capacity to provide adequate characterizations for the GE distribution with an explicit solution.

\section{Acknowledgments}

Thanks go to all staff of Department of Mathematics, Faculty of Science, University of Lagos, Nigeria and to all the supervisors of the corresponding author for their intellectual supports.

\section{References}

1. G. M.Cordeiro, E. M. M. Ortega, S. Nadarajah, The Kumaraswany weibull distribution with application to failure data, J.Franklin Inst. 347 (2010) 1399-1429.

2. M. H.Tahir, M. A. Hussain, G. M. Cordeiro, M. El-Morshedy and M. S. Eliwa, A new Kumaraswamy generalized family of distributions with properties, applications, and bivariate extension, Mathematics. 8 (2020) $1-28$.

3. A. Alzaatreh, F. Famoye, and C. Lee. Gamma-Pareto distribution and its applications, J. Mod Appl. Stat. Methods, 11 (2012) 78-94.

4. A. Alzaatreh, C. Lee and F. Famoye, Weibull-Pareto distribution and its applications, Commun. Stat. Theory Methods, 42 (2013) 1673-1691.

5. M. Bouguignon, R. B. Silva, L. M. Zea and G. M. Cordeiro, The Kumaraswamy-pareto distribution, J. Stat. Theory Appl. 12 (2013) 129144.

6. S. B. Chhetri, A. A. Akinsete, G. Aryal and H. Long, Kumaraswamy transmuted pareto distribution, J. Stat. Distrib. Appl. 4:11 (2017) 1-24.

7. E. E. E. Akarawak, I. A. Adeleke and R. O. Okafor, The weibull-rayleigh distribution and its properties, J. Eng. Res. 18 (2013) 56-67.

8. F. Famoye, E. E. E. Akarawak and M. Ekum. Weibull-normal distribution and its applications, J. Stat. Theory Appl. 17 (2018) 4-12.

9. U. Akata and J. E. Osemwenkhae, The weibull logistic-exponential distribution: its properties and applications, Earthline J. Math. Sci. 5 (2020) 197-216.

10. A. A. Al-Kadim and M. A Boshi, Exponential Pareto distribution, Math. theory model., 3 (2013) 135-146.

11. A. Luguterah, and S. Nasiru, Transmuted exponential Pareto distribution, Far East J. Theor. Stat. 50 (2015) 31-49.

12. I. Elbatal and G. Aryal, A new generalization of the exponential distribution, J. Inf. Optim. Sci. 38 (2017) 675-697.

13. H. Salem, Exponentiated exponential Pareto distribution: properties and estimation, Adv. \& Appl. in Stat. 57 (2019) 89-104.

14. G. Aryal, On the beta exponential Pareto distribution, Stat. Optim. Inf. Comput. 7 (2019) 417-438

15. N. I .Rashwan and M. M. Kamel, The beta exponential Pareto distribution, Far East J. Theor. Stat. 58 (2020) 91-113.

16. B. Gompertz, On the nature of the function of expressive of the law of human mortality and on new mode of determining the value of life contingencies, Philos. Trans. R. Soc. Lond., B, Biol. Sci. 182 (1824) 513-585.

17. T. G. leren, F. M. Kromtit, B. U. Agbor, I. B. Eraikhuemen and P. P. Koleoso, A power Gompertz distribution: model, properties and application to bladder cancer data, Asian Res. J. Math. 15 (2019) 1-14. 
18. M. Alizadeh, G. M. Cordeiro, P. L. G. Bastos and I. Ghosh, The Gompertz-G family of distributions. J Stat Theory Pract 11 (2017) 179207.

19. P. E. Oguntunde, M. A. Khaleel, H. I. Okagbue, A. A. Opanuga and F. O. Owolabi, The Gompertz inverse exponential (GoIE) distribution with Application, Cogent math. Stat. (2018) 1-17.

20. J. A. Adewara, J. S. Adeyeye, The comparative study of Gompertz exponential distribution and other three parameter distributions of exponential class, CJPL. 8 (2020) 1-13.

21. P. E. Oguntunde, M. A. Kheleel, T. A. Mohammed and I. O.Hilary, The Gompertz frechet distribution: properties and applications, Cogent math Stat. 6 (2019) 1-12.

22. P. O. Koleoso, A. U. Chukwu and T. A. Bamiduro, A three parameter Gompertz lindley distribution: its properties and applications, Math. theory model., 9 (2019) 29-42.

23. M. A. Khaleel, P. E. Oguntunde, M. T. Ahmed and N. O. Ibrahim, The Gompertz flexible weibull distribution and its applications, Malays. J. Math. Sci., 14 (2020) 169-190.

24. J. T. Eghwerido, L. Nzei, I. David and O. Adubisi, The gompertz extended generalized exponential distribution: properties and applications, Commun. Fac. Sci. Univ. Ank. Ser. A1 Math. Stat., 69 (2020) 739-753.

25. P. E. Oguntunde, M. A. Khaleel, M. T. Ahmed, A. O. Adejumo and O. A. Odetunmibi, A new generalization of the Lomax distribution with increasing, decreasing and constant failure rate, Model. Simul. Eng., 2017 (2017) 1-6.

26. J. T. Eghwerido, S. C. Zelibe and E. Efe-Eyefia, Gompertz-alpha power inverted exponential distribution: properties and applications, Thailand Stat., 18 (2020) 319-332.

27. F. B. Mohammed, K. A. Manju, U. K. Abdullahi, M. M. Sani and S. Kuje, A study of some properties and goodness of -fit of a Gompertzrayleigh mode, Asian J. Probab. Stat, 9 (2020) 18-31.

28. A. A. Ogunde, G. A. Olalude and D. O. Omosigho, The Gompertz gumbel II distribution : properties and applications, Acad. J. Appl. Math. Sci.. 7 (2021) 1-15.

29. H. A. David and H. N. Nagaraja. Oder statistics. John Wiley, NY. 2003.

30. B. C. Arnold, N. Balakrishnan and N. H. Nagaraja. A first course in order statistics. John Wiley, New York 1992.

31. P. R. D. Marinho, M. Bourguignon and C. R. B. Dias. R-packageadequacy model. https://cran.rproject.org/web/packages/AdequacyModel//AdequacyModel.pdf.

32. E. T. Lee and J. Wang, Statistical methods for survival data analysis. Wiley, New York, 2003.

33. I. Elbatal, and H. Z. Muhammed, Exponentiated generalized inverse weibull distribution, Appl. Math. Sci. 8 (2014) 3997-4012.

34. A. Ateeq, T. B. Qasim and A. R. Alvi, An extension of Rayleigh distribution and applications, Cogent math. Stat. 6 (2019) 1-16. 